\title{
A
}

Acta HealthMedica

Volume 2, Issue 2, April-June 2017, Pages: 177, DOI: http://dx.doi.org/10.19082/ah177

\section{CANCER REGISTRY SYSTEM IN NIGERIA: A QUANTITATIVE STUDY BASED ON WHO ICTRP STANDARDS}

\author{
Yusif Sulieman ${ }^{1}$, Sharareh R. Niakan Kalhori ${ }^{2}$
}

1: MSc Student of Health Information Technology, Department of Health Information Management, School of Allied Medical Sciences, Tehran University of Medical Sciences, Tehran, Iran.

2: Assistant Professor of Medical Informatics, Department of Health Information Management, School of Allied Medical Sciences, Tehran University of Medical Sciences, Tehran, Iran.

\section{Correspondence:}

Sharareh R. Niakan Kalhori, Tel: +98.21- 88982886, Fax:+98.21-88983025, E-mail: sh-rniakank@sina.tums.ac.ir

\section{TYPE OF ARTICLE: CONFERENCE ABSTRACT}

\begin{abstract}
Introduction: Nigeria, among the first African countries to establish cancer registry, began in the 1960's, at the Pathology Department of the University College Hospital, Ibadan in the south-west of the country. Lack of higher quality data in regard to chronic disease is making it difficult for policy makers to analyze and evaluate trend and burden of disease. The cancer registry is a tool to access and monitor patients' data, and control the information for assessment and strategic planning in a specific population in a time frame. The weak function of cancer surveillance at national level, might be due to a nonstandard cancer registry system. There is a standard introduced by the World Health Organization (WHO) called ICTRP (International Clinical Trials Registry Platform) which is efficient for both clinical and research purposes data management. Here, we have used this standard to assess African cancer registries in order to understand how useful the available systems are for patients, policy makers, clinicians and researchers.

Methods: Based on cancer reports in Nigeria from 2009 through 2013, released in 2016 by the Nigerian Federal Ministry of Health and available since June 2016 (http://nigeriancancerregistries.net/ accessed in 23/12/2016), we assessed the system registration according to ICTRP main standards.

Results: Out of nine main standards including content, accessibility, quality and validity, unambiguous identification, technical capacity, administration and governance, partner registries and data interchange standards of ICTRP, only two standards were considered, including content, and accessibility.

Conclusion: Cancer registry system development based on available standards, may ensure all users to obtain the best expected benefits according to their needs. Defected systems only create more workload and cost with less efficiency and effectiveness.

KEYWORDS: Cancer, Registry, Standards, ICTRP
\end{abstract}

\footnotetext{
Abstracts of First National Congress of Medical Informatics, Mashhad, Iran, February 2017

(C) 2017 The Authors. This is an open access article under the terms of the Creative Commons Attribution-NonCommercialNoDerivs License, which permits use and distribution in any medium, provided the original work is properly cited, the use is non-commercial and no modifications or adaptations are made.
} 